\title{
A Nuclease-Hypersensitive Region Forms De Novo after Chromosome Replication
}

\author{
MARK J. SOLOMON† AND ALEXANDER VARSHAVSKY* \\ Department of Biology, Massachusetts Institute of Technology, Cambridge, Massachusetts 02139
}

Received 11 May 1987/Accepted 14 July 1987

\begin{abstract}
Regular nucleosome arrays in eucaryotic chromosomes are punctuated at specific locations, such as active promoters and replication origins, by apparently nucleosome-free sites, also called nuclease-hypersensitive, or exposed, regions. The $\sim \mathbf{4 0 0}$-base-pair-exposed region within simian virus 40 (SV40) chromosomes is present in approximately $20 \%$ of the chromosomes in lytically infected cells and encompasses the replication origin, transcriptional enhancer, and both late and early SV40 promoters. We report that nearly all SV40 chromosomes lacked the exposed region during replication and that newly formed chromosomes acquired the exposed region to the same degree as did bulk SV40 chromosomes within $1 \mathrm{~h}$ after replication. Furthermore, a much lower but significant level of exposure was detectable in late SV40 replication intermediates, indicating that formation of the exposed region could start within minutes after passage of the replication fork.
\end{abstract}

We detected the presence of the exposed region within simian virus $40(8,10,17,18,21,22,25)$ by using the ability of the restriction endonuclease HaeIII to selectively excise a 325-base-pair replication origin-proximal DNA fragment from isolated simian virus 40 (SV40) chromosomes that had been fixed with formaldehyde $(17,22)$. The use of this nonkinetic assay, in which both limit fixation and limit digestion conditions were used, permitted precise quantitation of the extent of exposure in both pulse-labeled and bulk SV40 chromosomes. To follow the postreplicative formation of the exposed region, SV40-infected CV-1 cells were pulselabeled with $\left[{ }^{3} \mathrm{H}\right]$ thymidine for $15 \mathrm{~min}$ followed by a chase in the presence of unlabeled thymidine (Fig. 1A). In a parallel experiment, SV40-infected cells were incubated with $\left[{ }^{3} \mathrm{H}\right]$ thymidine for $18 \mathrm{~h}$ to allow subsequent determination of the bulk level of exposure (Fig. 1A, lane d). SV40 chromosomes were isolated, fixed with formaldehyde, and digested with HaeIII to determine the fraction of labeled chromosomes containing the exposed region.

SV40 chromosomes containing the exposed region were already present by $15 \mathrm{~min}$ of chase (Fig. 1A). Their proportion increased until $60 \mathrm{~min}$ of chase and declined slightly throughout $8 \mathrm{~h}$. From comparison with marker lanes a and b in Fig. $1 \mathrm{~A}$, containing respectively 25 and $5 \%$ as much ${ }^{3} \mathrm{H}$ as the experimental lanes did, we estimate that approximately $15 \%$ of the labeled SV40 chromosomes contained the exposed region by $1 \mathrm{~h}$ of chase. The extent of exposure was quantitatively reproducible within single experiments but varied slightly between experiments, most likely because of the strong temperature dependence of the exposed region formation in this SV40 strain (1). We consistently observed a slight decline in exposure after $1 \mathrm{~h}$ of chase, perhaps due to recruitment of a subset of chromosomes into virions, which lack the exposed region $(1,12)$.

A shorter labeling period $(5 \mathrm{~min})$ and shorter times of chase were used to examine earlier postreplicative times (Fig. 1B). Only a trace of pulse-labeled SV40 chromosomes (undetectable at this fluorographic sensitivity) contained the exposed region immediately after the 5-min pulse (Fig. 1B,

\footnotetext{
* Corresponding author.

† Present address: Department of Biochemistry and Biophysics, University of California, San Francisco, CA 94143.
}

lane 0 ). The extent of exposure increased approximately linearly through $55 \mathrm{~min}$ of chase. The final level of approximately $35 \%$ exposure was slightly higher than the extent of exposure observed in chromosomes continuously labeled for $18 \mathrm{~h}$ (Fig. 1B, lane d). To compare directly the extent of exposure in labeled versus bulk SV40 chromosomes, we electrophoresed samples from the same HaeIII digests as for Fig. 1B in an agarose gel alongside dilutions of deproteinized, HaeIII-digested DNA derived from the same pulsechase samples. Southern hybridization of the resulting patterns with the ${ }^{32} \mathrm{P}$-labeled 325-base-pair HaeIII fragment that is contained within the exposed region showed that approximately $25 \%$ of the bulk SV40 chromosomes contained the exposed region at all times of chase (Fig. 1C, lanes s). Thus, the conditions of high specific radioactivity labeling did not perturb the extent of exposure in bulk SV40 chromosomes.

The above results suggest that although newly replicated chromosomes acquire the bulk level of exposure during the first hour postreplication, formation of the exposed region in a small proportion of SV40 chromosomes can start within minutes after passage of the replication fork. To examine the extent of exposure in replication intermediates, we labeled SV40-infected cells with $\left[{ }^{3} \mathrm{H}\right]$ thymidine for $20 \mathrm{~min}$, extracted the SV40 chromosomes, and fixed them with formaldehyde. Fixed chromosomes were sedimented in a sucrose gradient to yield five sets of pooled fractions containing either fully replicated chromosomes (Fig. 2A, lane e) or various replication intermediates (Fig. 2A, lanes a through d). Pulselabeled replication intermediates should contain relatively little label in replication terminus-proximal regions (4). To verify that this requirement was met, we purified the DNA from each of the five pools mentioned above, digested it with HaeIII, and analyzed it by gel electrophoresis (Fig. 2C). As expected, replication intermediates contained relatively little label in the three DNA fragments that mapped at or close to the replication terminus (Fig. 2C, arrowheads; compare especially lanes $a$ and $b$ with lane e). Since replication fork movement slows down during the final stages of replication and pauses at the latest Cairns structure stage $(6,20)$, the pulse-labeled replicating chromosomes (Fig. 2A) are enriched in late replication intermediates. Figure $2 \mathrm{~B}$ shows that only 1 to $2 \%$ of these replication intermediates (lanes a and b) and 3 to $4 \%$ of the newly segregated chromosomes (lanes 


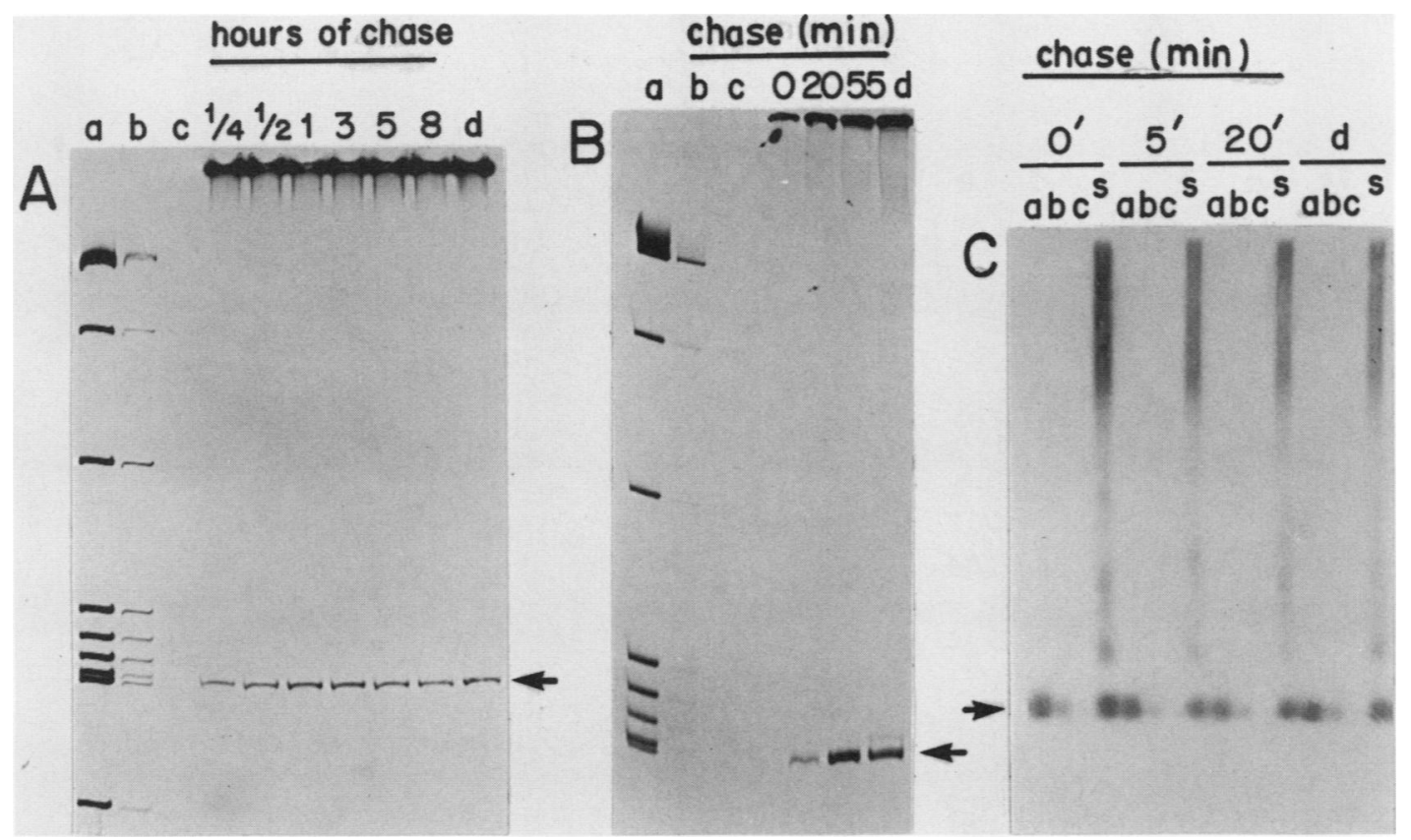

FIG. 1. Postreplicative formation of the SV40 exposed region. African green monkey CV-1 cells were infected with SV40 (strain 777 ) and labeled with [methyl ${ }^{3} \mathrm{H}$ ]thymidine $(>80 \mathrm{Ci} / \mathrm{mmol})$ at $0.5 \mathrm{mCi} / \mathrm{ml}(19,20)$ for either $15 \mathrm{~min}(\mathrm{~A})$ or $5 \mathrm{~min}(\mathrm{~B}$ and $\mathrm{C})$, followed by a cold chase $(19,20)$ for either $0.25,0.5,1,3,5$, or $\mathbf{~} \mathrm{h}(\mathrm{A})$ or for 0,20 , or $55 \mathrm{~min}$ (B and C), as indicated. For the samples in lanes d, the labeling (at a 50 -fold-lower concentration of [methyl $-{ }^{3} \mathrm{H}$ ] thymidine) continued for $18 \mathrm{~h}$. Labeling and chase regimens were designed so that all samples of SV40 chromosomes were isolated at $40 \mathrm{~h}$ postinfection. SV40 chromosomes were isolated as described previously (22) except that $N$-2-hydroxyethylpiperazine- $N^{\prime}$-2-ethanesulfonic acid (HEPES) was substituted for Tris in the extraction buffer. For panel A, SV40 chromosomes were additionally purified by sedimentation in a sucrose gradient $(19,20,23)$. Chromosomes were then fixed in $1 \%$ formaldehyde as previously described $(17,22)$, extensively dialyzed (17), limit-digested with HaeIII, and electrophoresed either in a $7 \%$ polyacrylamide gel (A and B) or in a $1.5 \%$ agarose gel $(C)(17,22)$. A 1-cm strip of $1 \%$ agarose was present on top of the polyacrylamide gels. Equal amounts of radioactivity were loaded onto each lane within each panel except for marker lanes a through $c$, which contained the products of a HaeIII digest of naked SV40 [ $\left.{ }^{3} \mathrm{H}\right] \mathrm{DNA}$ from the same experiment. Lanes a through c contained 25,5 , and $1 \%$, respectively, of the radioactivity applied to the other lanes in the same panels. Detection was either by fluorography of $\left[{ }^{3} \mathrm{H}\right] \mathrm{DNA}$ in a polyacrylamide gel (A and B) or by Southern hybridization (C) to a 325-base-pair origin-proximal HaellI fragment that was contained within the exposed (nuclease-hypersensitive) region of SV40 chromosomes $(17,20)$. The lanes marked s (sample) in panel C contained formaldehyde-fixed, HaeIII-digested SV40 chromosomes.

d and e) contained the exposed region. Thus, although the steady-state extent of exposure (20 to 25\%) was reached gradually during approximately $1 \mathrm{~h}$ after chromosome replication (Fig. 1B), the exposed region formed within minutes of passage of the replication fork in a small proportion of chromosomes (Fig. 2B).

Our results show that formation of the exposed region in newly replicated SV40 chromosomes occurs continuously from a few minutes to an hour after replication. These findings are in general agreement with earlier qualitative analyses of the relationship between replication and formation of the SV40 exposed region. The greater sensitivity of the present experiments allowed detection of a small degree of exposure in replicating SV40 chromosomes, whereas a previous study from this laboratory detected none (23). Recent electron microscopic evidence (16) also suggests the presence of the exposed region in a small proportion of replicating SV40 chromosomes, in agreement with our biochemical results.

The exposed region has been operationally defined as the DNA that is selectively excised by a restriction endonuclease from formaldehyde-fixed chromosomes as naked DNA. The formaldehyde assay detects the exposed region within SV40 chromosomes fixed either in vitro (22) or in vivo (in intact cells) (17). This assay produced one of the first identifications of the exposed region in $\operatorname{SV} 40(15,21,22)$ and has since been used to detect exposed regions in other chromosomes such as the Saccharomyces cerevisiae $2 \mu \mathrm{m}$ plasmid (11). Although formaldehyde efficiently cross-links histones to DNA both in vitro and in vivo, it completely fails to cross-link a variety of other DNA-binding proteins to their cognate DNA sequences in vitro (17). Thus, although the SV40 exposed region lacks formaldehyde-cross-linkable proteins (Fig. 1 and 2) $(17,22)$, it certainly contains DNA-bound proteins in vivo $(5,7,25)$.

Our finding that the SV40 exposed region formed largely postreplicatively, with a measurable (and for many chromosomes, a considerable) delay, is an argument against direct templating mechanisms, in which a preexisting exposed region would survive the passage of the replication fork and nucleate formation of the second exposed region in the daughter chromosomes. Thus, either replication is confined to those SV40 chromosomes that initially lack the exposed region, or the passing replication fork erases the preexisting exposed region. In either case, the subsequent appearance of the exposed region requires a de novo assembly process involving trans-acting factors. This model is consistent with the recent evidence that passage of an SV40 replication fork in vitro displaces an otherwise stable and active transcription complex assembled on a Xenopus 5S RNA gene $(2,26)$. Furthermore, several lines of recent evidence suggest that exposed regions can form de novo in the absence of DNA 


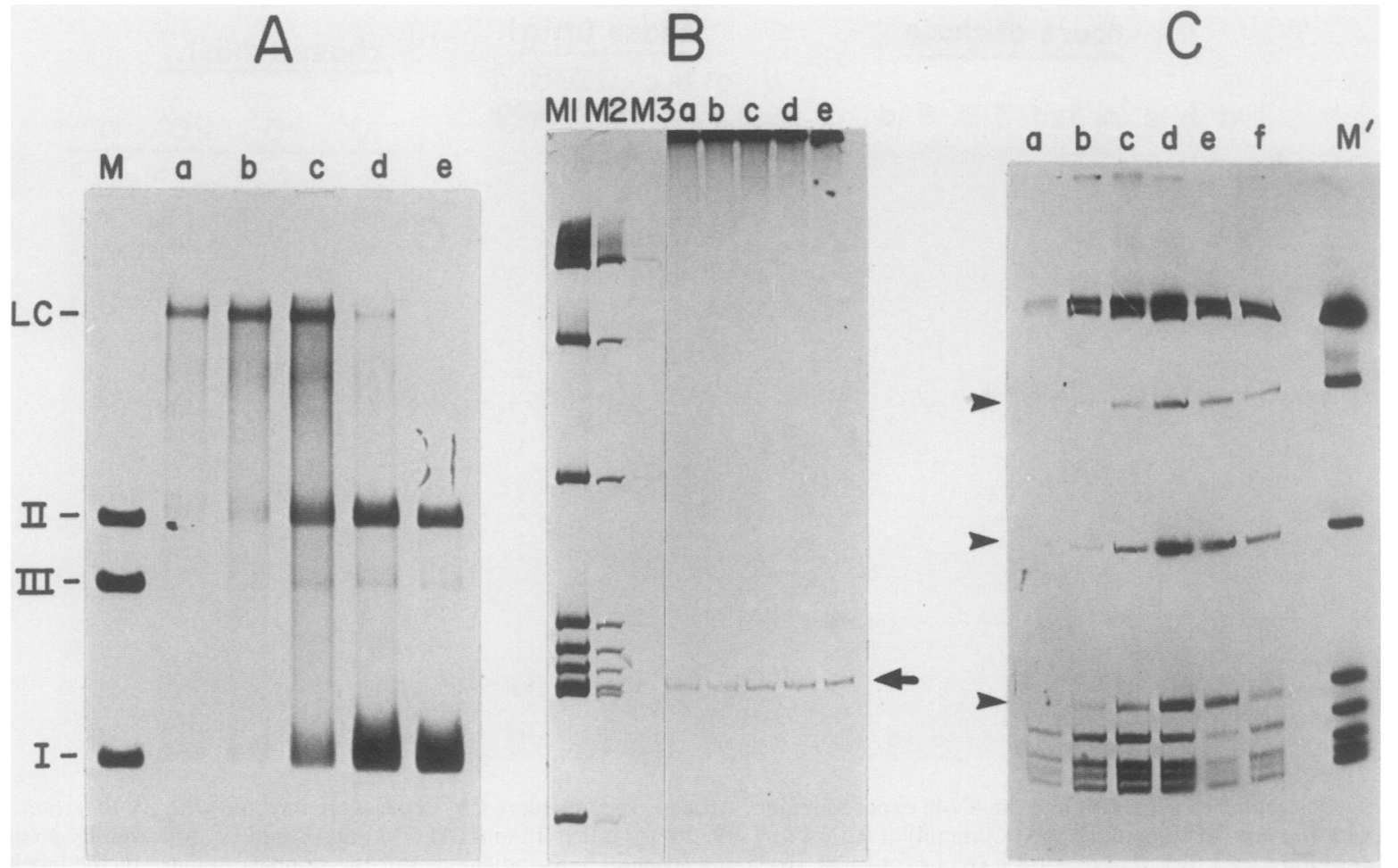

FIG. 2. Detection of the SV40 exposed region in replication intermediates. SV40-infected cells were labeled with $\left[\right.$ methyl- $\left.{ }^{3} \mathrm{H}\right]$ thymidine for $20 \mathrm{~min}$ at $40 \mathrm{~h}$ postinfection. Isolated SV40 chromosomes were fixed with formaldehyde $(17,22)$ and thereafter were centrifuged through a 5 to $30 \%$ sucrose gradient containing $0.1 \mathrm{M} \mathrm{NaCl}, 1 \mathrm{mM} \mathrm{Na}$-EDTA, $0.5 \mathrm{mM}$ Na-ethylene glycol-bis( $\beta$-aminoethyl ether)- $N, N, N^{\prime}, N^{\prime},-$ tetracetic acid (EGTA), and $10 \mathrm{mM}$ Na-HEPES (pH 7.5) in an SW41 rotor (Beckman) at 35,000 rpm for 150 min. Lanes a through e for all panels contained pooled pairs of gradient fractions from near the bottom of the sucrose gradient (a) through the peak of mature chromosomes (e). (A) Deproteinized DNA (17) electrophoresed in an agarose gel. Lane M (markers) contained closed circular (I), nicked circular (II), and linear (III) SV40 DNA. LC denotes the latest Cairns structure $(19,20,23)$. (B) Equal amounts of radioactivity from each pool in panel A were limit-digested with $\mathrm{HaeIII}$ and electrophoresed in a 7\% polyacrylamide gel. Lanes M1 through M3 (deproteinized [17], HaeIII-digested total SV40 $\left[{ }^{3} \mathrm{H}\right]$ DNA from the same experiment) contained 25,5 , and $1 \%$, respectively, of the total radioactivity applied to lanes a through e. The arrow denotes the 325-base-pair HaellI fragment contained within the exposed region in SV40 chromosomes. (C) Chromosome samples from each pool were deproteinized (17), limit-digested with HaeIII, and electrophoresed in a polyacrylamide gel. Arrowheads indicate the (terminus-proximal) fragments that are underrepresented in pools of SV40 replication intermediates. Lanes: f, a HaeIII digest of DNA purified from the initial formaldehyde-fixed chromosome sample before sedimentation in a sucrose gradient; $\mathrm{M}^{\prime}$, a HaeIII digest of uniformly ${ }^{3} \mathrm{H}$-labeled SV40 DNA. Samples were electrophoresed either in a $1 \%$ agarose gel (A) or in a $7 \%$ polyacrylamide gel (B and C). [ $\left.{ }^{3} \mathrm{H}\right] \mathrm{DNA}$ in gels was detected by fluorography.

replication $(3,24)$. An earlier observation that an exposed region, once induced in a cellular gene, can propagate indefinitely in the absence of the inducing factor (9) is also not inconsistent with the above model, since a delay in the reappearance of the exposed region after DNA replication would not have been detected by the assay used.

Although a number of faithful in vitro transcription and replication systems have been developed in recent years, a satisfactory in vitro system in which a well-defined exposed region could form within a preexisting nucleosome array is still to be devised. Thus, we know little about the process by which preexisting nucleosomes are either modified or displaced at a specific site with concurrent assembly of DNAbound protein complexes to yield an exposed region. Understanding the detailed pathway of exposed-region formation, either after passage of a replication fork or within nondividing cells, is essential for deeper insights into both the dynamic aspects of chromatin structure and the mechanisms of epigenetic inheritance.

We thank Daniel Finley and John McGrath for their comments on the manuscript and Barbara Doran for secretarial assistance.

This work was supported by Public Health Service grant CA43309 to A.V. from the National Cancer Institute. M.J.S. was supported by a predoctoral fellowship from the National Science Foundation.

\section{LITERATURE CITED}

1. Ambrose, C., V. Blasquez, and M. Bina. 1986. A block in initiation of simian virus 40 assembly results in the accumulation of minichromosomes containing an exposed regulatory region. Proc. Natl. Acad. Sci. USA 83:3287-3291.

2. Bedinger, P., M. Hochstrasser, C. V. Jongeneel, and B. M. Alberts. 1983. Properties of the T4 bacteriophage DNA replication apparatus: the T4 dda DNA helicase is required to pass a bound RNA polymerase molecule. Cell 34:115-123.

3. Chiu, C.-P., and H. M. Blau. 1984. Reprogramming cell differentiation in the absence of DNA synthesis. Cell 37:879-887.

4. Danna, K. J., and D. Nathans. 1972. Bidirectional replication of simian virus 40 DNA. Proc. Natl. Acad. Sci. USA 69:30973100.

5. Davidson, I., C. Fromental, P. Augereau, A. Wildeman, M. Zenke, and P. Chambon. 1986. Cell-type specific protein binding to the enhancer of simian virus $\mathbf{4 0}$ in nuclear extracts. Nature (London) 323:544-548.

6. DePamphilis, M. L., L. E. Chalifour, M. F. Charette, M. E. Cusick, R. T. Hay, E. A. Hendrickson, C. G. Pritchard, L. C. Tack, P. M. Wassarman, D. T. Weaver, and D. O. Wirak. 1983. Papovavirus chromosomes as a model for mammalian DNA 
replication, p. 423-447. In N. R. Cozzarelli (ed.), Mechanisms of DNA replication and recombination. Alan R. Liss Inc., New York.

7. Dynan, W. S., and R. Tjian. 1983. The promoter-specific transcription factor $\mathrm{Spl}$ binds to upstream sequences in the SV40 early promoter. Cell 35:79-87.

8. Eissenberg, J. C., I. L. Cartwright, G. H. Thomas, and S. C. R. Elgin. 1985. Selected topics in chromatin structure. Annu. Rev. Genet. 19:485-536.

9. Groudine, M., and H. Weintraub. 1982. Propagation of globin DNAase I-hypersensitive sites in absence of factors required for induction: a possible mechanism for determination. Cell 30:131139.

10. Jakobovitz, E. B., S. Bratosin, and Y. Aloni. 1980. A nucleosome-free region in SV40 minichromosomes. Nature (London) 285:263-265.

11. Livingston, D. M. 1982. A sequence of the yeast $2 \mu \mathrm{m}$ DNA plasmid chromosome near the origin of replication is exposed to restriction endonuclease digestion. J. Mol. Biol. 160:397-410.

12. Milavetz, B. 1986. Analysis of the origin-specific nucleosomefree region in SV40 encapsidation intermediates. Virology 153:310-313.

13. Pederson, D. S., F. Thoma, and R. T. Simpson. 1986. Core particle, fiber, and transcriptionally active chromatin structure. Annu. Rev. Cell Biol. 2:117-147.

14. Saragosti, S., G. Moyne, and M. Yaniv. 1980. Absence of nucleosomes in a fraction of SV40 chromatin between the origin of replication and the region coding for the late leader RNA. Cell 20:65-73.

15. Scott, W. A., and D. J. Wigmore. 1978. Sites in SV40 chromatin which are preferentially cleaved by endonucleases. Cell 15: 1511-1518.

16. Sogo, J. M., H. Stahl, T. Koller, and R. Knippers. 1986. Structure of replicating simian virus $\mathbf{4 0}$ minichromosomes: the replication fork, core histone segregation and terminal structures. J. Mol. Biol. 189:189-204.
17. Solomon, M. J., and A. Varshavsky. 1985. Formaldehydemediated DNA-protein crosslinking: a probe for in vivo chromatin structures. Proc. Natl. Acad. Sci. USA 82:6470-6474.

18. Sundin, O., and A. Varshavsky. 1979. Staphylococcal nuclease makes a single non-random cut in the simian virus 40 viral minichromsome. J. Mol. Biol. 132:535-546.

19. Sundin, O., and A. Varshavsky. 1980. Terminal stages of SV40 DNA replication proceed via multiply intertwined catenated dimers. Cell 21:103-114.

20. Sundin, O., and A. Varshavsky. 1981. Arrest of segregation leads to accumulation of highly intertwined catenated dimers: dissection of the final stages of SV40 DNA replication. Cell 25:659-669.

21. Varshavsky, A., O. H. Sundin, and M. J. Bohn. 1978. SV40 viral minichromosome: preferential exposure of the origin of replication as probed by restriction endonucleases. Nucleic Acids Res. 5:3469-3477.

22. Varshavsky, A., O. H. Sundin, and M. Bohn. 1979. A stretch of late SV40 viral DNA about 400 bp long which contains the origin of replication is specifically exposed in SV40 minichromosomes. Cell 16:453-466.

23. Varshavsky, A., O. Sundin, E. Özkaynak, R. Pan, M. J. Solomon, and R. Snapka. 1983. Final stages of DNA replication: multiply intertwined catenated dimers as SV40 segregation intermediates, p. 463-494. In N. R. Cozarelli (ed.), Mechanisms of DNA replication and recombination. Alan R. Liss Inc., New York.

24. Weintraub, H. 1985. Assembly and propagation of repressed and derepressed chromosomal states. Cell 42:705-711.

25. Weiss, E., D. Ghose, P. Schultz, and P. Oudet. 1985. T-antigen is the only detectable protein on the nucleosome-free origin region of isolated simian virus 40 minichromosomes. Chromosoma 92:391-400.

26. Wolffe, A. P., and D. D. Brown. 1986. DNA replication in vitro erases a Xenopus 5S RNA gene transcription complex. Cell 47:217-227. 